\title{
Persistent Hiccups Following Use of Oral Dexamethasone: A Report of Two Cases from Abakaliki, Nigeria
}

\author{
Chukwuemeka O. Eze*, Thomas O. Nnaji, Monday U. Nwobodo \\ Internal Medicine Department, Alex Ekwueme Federal University Teaching Hospital Abakaliki (AEFUTHA), Ebonyi State, Nigeria \\ Email: *drezeconauth@yahoo.com, *drezeconauth@gmail.com
}

How to cite this paper: Eze, C.O., Nnaji, T.O. and Nwobodo, M.U. (2020) Persistent Hiccups Following Use of Oral Dexamethasone: A Report of Two Cases from Abakaliki, Nigeria. Case Reports in Clinical Medicine, 9, 282-287.

https://doi.org/10.4236/crcm.2020.99040

Received: August 10, 2020

Accepted: September 14, 2020

Published: September 17, 2020

Copyright $\odot 2020$ by author(s) and Scientific Research Publishing Inc. This work is licensed under the Creative Commons Attribution International License (CC BY 4.0).

http://creativecommons.org/licenses/by/4.0/

\begin{abstract}
Hiccups are common, usually mild with no obvious cause and often resolve spontaneously. They are classified as transient, persistent and intractable depending on the duration. Drug induced hiccups have been reported in medical literature but not common and corticosteroids are often cited. This report involved 2 male patients who developed persistent hiccups following use of oral dexamethasone for inflammatory conditions. The hiccups were severe and intolerable and could not stop despite use of metoclopramide and chlorpromazine. The hiccups only stopped following discontinuation of the dexamethasone. It should be noted that low dose of dexamethasone was used. Management of dexamethasone induced hiccups involves discontinuation of the drug, steroid rotation if the patient is steroid dependent and use of metoclopramide and chlorpromazine. Clinicians should be aware of this known but rare adverse effect of dexamethasone as it could be severe, distressful and negatively impart patients care. There is a need for a high index of suspicion whenever a patient develops hiccups while taking dexamethasone.
\end{abstract}

\section{Keywords}

Hiccups, Dexamethasone, Abakaliki, Nigeria

\section{Introduction}

Hiccups are sudden, uncontrolled contractions of the diaphragm, followed by immediate inspiration and closure of the glottis over the trachea [1]. They are also known as Singulta. Hiccup or singultus is derived from the Latin word singult, which means "the act of catching one's breath while sobbing" [2]. They are very common, usually mild with no obvious cause and often resolve sponta- 
neously. They occasionally become severe, prolonged and distressful.

Hiccups are classified depending on the duration into acute/transient (less than 48 hours), persistent (longer than 48 hours) and intractable (longer than 1 month) [3] [4]. The self limited episodes of hiccups (transient) are common in healthy individuals and believed to be induced by the rapid stomach distension and irritation caused by overeating, eating too fast, ingesting spicy food, drinking carbonated drinks, aerophagia and sudden change in ingested food temperature [5]. Persistent hiccups are most likely to be associated with an underlying pathological, anatomic or organic disease process [6]. Intractable hiccups are usually indicative of a serious organic disturbance and if left untreated, can cause severe discomfort, depression, reduced physical strength, and even death [7] [8].

The aetiology of hiccups is largely unknown, but the neural pathway consists reflex arc of 3 components, the afferent limb including phrenic, vagus and sympathetic nerves to convey somatic and visceral sensory signals, the central processing unit in the midbrain and the efferent limb traveling in motor fibers of phrenic nerves to diaphragm and intercostal nerves to the intercostal muscles, respectively [9] [10] [11]. Dopaminergic and gamma-amino-butyric-acid (GABAergic) neurotransmitters modulate this central mechanism. Activation of above pathway by chemical, mechanical and psychological stimuli results in hiccups. It has been proposed that dexamethasone decreases the threshold for synaptic transmission in the midbrain and eventually induces hiccups [12].

Several medical conditions have been associated with the development of hiccups and they include gastrointestinal, neurological, pulmonary, psychogenic, cardiovascular, metabolic, anesthesia related and drug induced conditions [13] [14]. Although steroid induced hiccups are rare, they are much more frequent with dexamethasone than with other corticosteroids [15] [16].

There has not been any reported case of dexamethasone induced hiccups in Nigeria despite its wide use in management of inflammatory conditions. It is against this background that we reported these 2 cases of persistent hiccups following use of oral dexamethasone.

\section{Case 1}

A 39-year-old male civil servant presented to the outpatient clinic with recurrent cough and nasal congestion of about 4 weeks. There was associated sneezing and mild frontal headache but there was no history of fever or hemoptysis. The above symptoms were worsened by exposure to cold environment. He is known to have atopy with previous history of cough and nasal congestion. Physical examination was essentially normal. His complete blood count was normal (Hemoglobin $14 \mathrm{~g} / \mathrm{dL}$, White blood cell count 4200/uL, Neutrophil 52\%, lymphocytes $45 \%$, Platelets $210,000 / \mathrm{uL}$ ). HIV serology was seronegative. COVID-19 test was also non-reactive. Radiological investigations were normal. He was placed on Levofloxacin and sinufed which gave him significant relief but symptoms recurred immediately the medications were stopped after 7 days. He was later 
given Dexamethasone (6 $\mathrm{mg}$ daily) and sinufed tablets. The cough, nasal congestion and headache subsided following use of above medications for 3 days but he developed persistent hiccups. He was given metoclopramide, $10 \mathrm{mg}$ thrice daily for two days. The hiccups persisted and worsened in severity as he became exhausted, had insomnia and couldn't tolerate oral feeds despite use of metoclopramide tablets. Chlorpromazine was later introduced and the dose was progressively escalated to $50 \mathrm{mg}$ thrice daily. The hiccups still persisted. At this point, drug induced hiccups were suspected and dexamethasone was eliminated from his medications. The hiccups stopped following the elimination of dexamethasone from his medications even when he didn't take chlorpromazine and a retrospective diagnosis of dexamethasone induced hiccups was made.

\section{Case 2}

A 65-year-old male trader presented to the outpatient clinic with progressive low back pains of about 5 years duration. The pain was progressive, left sided and radiated to the left thigh, leg and foot. There was associated difficulty with ambulation, insomnia and the pain was not relieved by use of over the counter medications. There were normal bowel and urinary functions. He was diagnosed to have type 2 diabetes in 2018 and is well-controlled on diet alone. He is not known to have any other chronic medical condition. Physical examination was normal except bilateral positive straight leg raising sign worse on the left. Radiological investigation suggested lumbosacral spondylosis with disc prolapse. Complete blood count and kidney function test were normal. He was commenced on tablets Diclofenac/Misoprostol $75 \mathrm{mg}$ bd and Gabapentin $300 \mathrm{mg}$ bd. After one week on above medications, the symptoms did not improve significantly. Tablets Celecoxib $200 \mathrm{mg}$ bd, Dihydrocodene $30 \mathrm{mg}$ bd, and Pregabalin $75 \mathrm{mg}$ bd were added to the previous medications. There was mild improvement of the symptoms but was limited by drowsiness. At 2 week review, the medications were adjusted to include Tablets Dexamethasone $4 \mathrm{mg}$ tds, Diclofenac/misoprostol $75 \mathrm{mg}$ bd, Celecoxib $200 \mathrm{mg}$ bd, Gabapentin $300 \mathrm{mg}$ bd and Omeprazole $20 \mathrm{mg}$ bd. Following the use of above medications for 2 days, the low back pain subsided significantly but he developed hiccups and worsening blood glucose control. A suspicion of dexamethasone induced hiccups was made and the dose was reduced to $2 \mathrm{mg}$ tds but the hiccups and hyperglycemia persisted. Dexamethasone was then discontinued after next 2 days and the hiccups and hyperglycemia resolved. He was then continued on the other medications with significant pain control.

\section{Discussion}

This study reported 2 cases of dexamethasone induced hiccups in Abakaliki Nigeria. It's surprisingly the first report of this known but rare side effect in Nigeria despite that Dexamethasone is widely available and frequently used in the management of inflammatory conditions. This suggests that the adverse effect is 
rare and/or that the clinicians have been generally unaware of the adverse effect. The above 2 cases were noted within a space of 4 weeks amongst two men. The male predilection in this study is similar to previously reported study [17]. The reason for male predilection could stern from higher male prevalence of the risk factors for hiccups [18].

Both of them developed persistent hiccups following use of oral dexamethasone for treatment of inflammatory conditions. The hiccups were severe and intolerable that they were distressed, exhausted and unable to sleep. They reported the side effect because it was severe, persistent and intolerable as their quality of life was significantly diminished. The hiccups persisted despite reduction of the dose of dexamethasone to $6 \mathrm{mg}$ daily. This suggests that the dexamethasone induced hiccups may not be dose related as previously reported [19].

The pharmacologic treatment of steroid induced hiccups, including steroid rotation [15], chlorpromazine, metoclopramide, haloperidol, and baclofen had been reported to stop hiccups [20]. Chlorpromazine and metoclopramide could not stop the hiccups in case 1 as it was drug induced. The author used hind sight of case 1 to discontinue the dexamethasone in case 2 without use of chlorpromazine and metoclopramide. Steroid rotation was not applied as the patients were not steroid dependent but rather the dexamethasone was discontinued.

If above interventions could not resolve the hiccups, non-pharmacologic treatment which involves the interruption of the vagal afferent limb of reflex arc or stimulating the vagal nerve was shown to successfully resolve intractable hiccups [20]. There is a need for future studies to determine the hospital prevalence and epidemiology of steroid induced hiccups and also investigate the potential biomarkers that can help identify susceptible individuals.

\section{Conclusion}

Dexamethasone has been reported to cause hiccups. Although hiccups are not usually life-threatening, they are important because they can be severe, intolerable and significantly diminish quality of life in patients. Discontinuation of dexamethasone or switching from dexamethasone to other corticosteroids has been reported to relieve hiccups. Dexamethasone should be used with caution due to this adverse effect.

\section{Acknowledgements}

We gratefully acknowledge Mrs. Nnenna Cindy EZE for typesetting and editing the article.

\section{Funding}

This work was funded by the Authors.

\section{Consent to Publish}

Written informed consent was obtained from the patients for their information 
to be published in anonymous form in this article.

\section{Authors' Contributions}

Chukwuemeka O EZE contributed to acquisition of data, analysis and interpretation of data and wrote the paper.

Monday U NWOBODO was involved in drafting and revising the manuscript. Thomas O NNAJI was involved in revising the manuscript.

\section{Conflicts of Interest}

The authors declare no conflicts of interest.

\section{References}

[1] Kolodzik, P.W. and Eilers, M.A. (1991) Hiccups (Singultus): Review and Approach to Management. Annals of Emergency Medicine, 20, 565-573. https://doi.org/10.1016/S0196-0644(05)81620-8

[2] Hiccup. http://en.wikipedia.org/wiki/Hiccup

[3] Hung, Y.M., Miller, M.A. and Patel, M.M. (2003) Persistent Hiccups Associated with Intravenous Corticosteroid Therapy. Journal of Clinical Rheumatology, 9, 306-309. https://doi.org/10.1097/01.rhu.0000089792.61349.0c

[4] Smith, H.S. and Busracamwongs, A. (2003) Management of Hiccups in the Palliative Care Population. American Journal of Hospice and Palliative Medicine, 20, 149-154. https://doi.org/10.1177/104990910302000214

[5] Chang, F.-Y. and Lu, C.-L. (2012) Hiccup: Mystery, Nature and Treatment. Journal of Neurogastroenterology and Motility, 18, 123-130.

[6] Cymet, T.C. (2002) Retrospective Analysis of Hiccups in Patients at a Community Hospital from 1995-2000. Journal of the National Medical Association, 94, 480-483.

[7] Vaidya, V. (2000) Sertraline in the Treatment of Hiccups. Psychosomatics, 41, 353-355. https://doi.org/10.1176/appi.psy.41.4.353

[8] Consults, D. Hiccups-Etilogy and Treatment. http://hslibrary.ucdenver.edu/

[9] Becker, D.E. (2010) Nausea, Vomiting, and Hiccups: A Review of Mechanisms and Treatment. Anesthesia Progress, 57, 150-156. https://doi.org/10.2344/0003-3006-57.4.150

[10] Howard, R.S. (1992) Persistent Hiccups. BMJ, 305, 1237-1238. https://doi.org/10.1136/bmj.305.6864.1237

[11] Hansen, B.J. and Rosenberg, J. (1993) Persistent Postoperative Hiccups: A Review. Acta Anaesthesiologica Scandinavica, 37, 643-646. https://doi.org/10.1111/j.1399-6576.1993.tb03781.x

[12] Lee, G.W., Oh, S.Y., Kang, M.H., Kang, J.H., Park, S.H., Hwang, I.G., Yi, S.Y., Choi, Y.J., Ji, J.H., Lee, H.Y. and Bruera, E. (2013) Treatment of Dexamethasone-Induced Hiccup in Chemotherapy Patients by Methylprednisolone Rotation. The Oncologist, 18, 1229-1234. https://doi.org/10.1634/theoncologist.2013-0224

[13] Fisher, C.M. (1967) Protracted Hiccup-A Male Malady. Transactions of the American Neurological Association, 92, 231-233.

[14] Takiguchi, Y., Watanabe, R., Nagao, K. and Kuriyama, T. (2002) Hiccups as an Adverse Reaction to Cancer Chemotherapy. Journal of the National Cancer Institute, 94, 772-774. https://doi.org/10.1093/jnci/94.10.772 
[15] Sugandhavesa, N., Sawaddiruk, P., Bunmaprasert, T., Pattanakuhar, S., Chattipakorn, S.C. and Chattipakorn, N. (2019) Persistent Severe Hiccups after Dexamethasone Intravenous Administration. American Journal of Case Reports, 20, 628-630. https://doi.org/10.12659/AJCR.915282

[16] Go, S.I., Koo, D.H., Kim, S.T., Song, H.N., Kim, R.B., Jang, J.S., Oh, S.Y., et al. (2017) Antiemetic Corticosteroid Rotation from Dexamethasone to Methylprednisolone to Prevent Dexamethasone-Induced Hiccup in Cancer Patients Treated with Chemotherapy: A Randomized, Single-Blind, Crossover Phase III Trial. The Oncologist, 22, 1354-1361. https://doi.org/10.1634/theoncologist.2017-0129

[17] Lee, G.W., Kim, R.B., Go, S.I., Cho, H.S., Lee, S.J., Hui, D., Bruera, E., et al. (2016) Gender Differences in Hiccup Patients: Analysis of Published Case Reports and Case-Control Studies. Journal of Pain and Symptom Management, 51, 278-283. https://doi.org/10.1016/j.jpainsymman.2015.09.013

[18] Eroglu, O. (2018) The Effect of Gender Differences in Protracted Hiccups. Nigerian Journal of Clinical Practice, 21, 1356-1360

[19] Cersosimo, R.J. and Brophy, M.T. (1998) Hiccups with High Dose Dexamethasone Administration: A Case Report. Cancer, 82, 412-414.

https://doi.org/10.1002/(SICI)1097-0142(19980115)82:2\%3C415::AID-CNCR23\%3E 3.0.CO;2-0

[20] Abbasi, A., Roque-Dang, C.M. and Malhotra, G.P.M.R. (2012) Persistent Hiccups after Interventional Pain Procedures: A Case Series and Review. $P M \& R, \mathbf{4}$, 144-151. https://doi.org/10.1016/j.pmrj.2011.09.005 